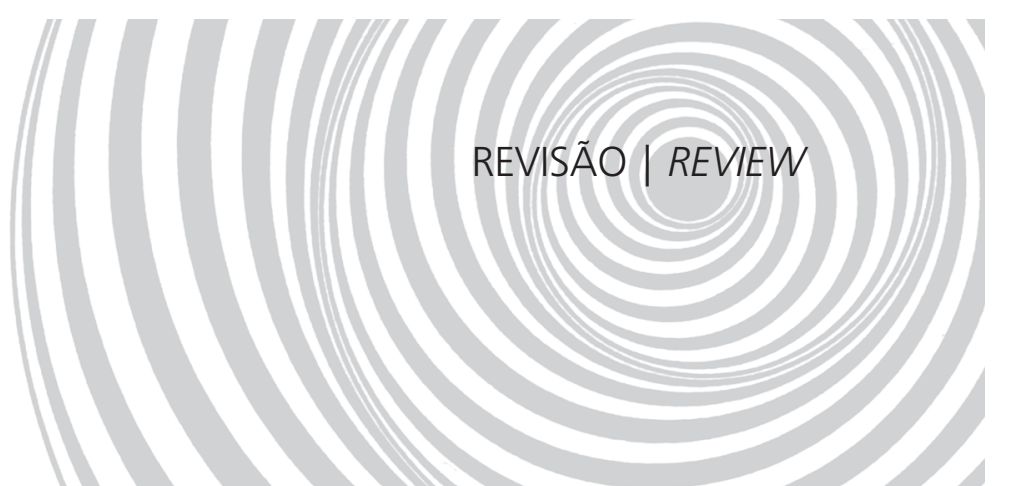

\title{
Terapia nutricional no diabetes gestacional
}

\section{Nutritional therapy in gestational diabetes}

Patricia de Carvalho PADILHA 1,2

Ana Beatriz SENA ${ }^{2}$

Jamile Lima NOGUEIRA ${ }^{2}$

Roberta Pimenta da Silva ARAÚJO3

Priscila Dutra ALVES²

Elizabeth ACCIOLY2

Cláudia SAUNDERS 2,4

RE S U M O

Trata-se de uma revisão da literatura científica sobre a terapia nutricional no Diabetes Mellitus Gestacional, sem restrição de data e com fontes primárias indexadas nas bases de dados SciELO, PubMed, Medline. Os resultados desta revisão apontam a intervenção nutricional como uma importante aliada no controle do Diabetes Mellitus Gestacional, trazendo potenciais benefícios à saúde materno-fetal. Na avaliação do estado nutricional materno devem ser empregados os indicadores antropométricos, dietéticos, bioquímicos, clínicos e funcional. Neste sentido, a avaliação dietética deve ser detalhada, com atenção para o fracionamento e composição das refeições, e grupos de alimentos presentes. No planejamento nutricional a distribuição de macronutrientes em relação ao consumo energético diário deve ser $45-65 \%$ de carboidratos, $15-20 \%$ de proteínas e $20-35 \%$ de lipídeos. Quanto a recomendação dos edulcorantes, são liberados para gestantes acesulfame K, aspartame, neotame, sacarina e sucralose. A atividade física também deve fazer parte da estratégia de tratamento do Diabetes Mellitus Gestacional, embora o impacto do exercício nas complicações neonatais ainda mereça ser rigorosamente testado. Ademais, estudos associam a habilidade de aconselhamento nutricional com a melhorara na adesão ao cuidado nutricional. Diante desses achados, para sucesso no controle do DMG são necessários: a participação da equipe inter e multidisciplinar, o cuidado pré-natal precoce, com assistência nutricional oportuna e a garantia da assistência de qualidade ao longo da gestação.

Termos de indexação: Cuidado pré-natal. Diabetes gestacional. Gestante. Nutrição.

\section{A B S T R A C T}

This is a scientific literature review about nutritional therapy in gestational diabetes mellitus, without date restriction and using the SciELO, PubMed and Medline databases. The results of this review show that nutritional

\footnotetext{
1 Universidade Federal do Rio de Janeiro, Instituto de Puericultura e Pediatria Martagão Gesteira, Serviço de Nutrição Clínica. R. Bruno Lobo, 50, Ilha do Fundão, 21920-612, Rio de Janeiro, RJ, Brasil. Correspondência para/Correspondence to: P.C. PADILHA. E-mail: <paticpadilha@yahoo.com.br>.

2 Universidade Federal do Rio de Janeiro, Instituto de Nutrição Josué de Castro, Programa de Pós-Graduação em Nutrição Departamento de Nutrição e Dietética. Rio de Janeiro, RJ, Brasil.

${ }^{3}$ Escola Nacional de Saúde Pública, Centro de Saúde. Rio de Janeiro, RJ, Brasil.

${ }^{4}$ Universidade Federal do Rio de Janeiro, Maternidade Escola. Rio de Janeiro, RJ, Brasil.
} 
96 | P.C. PADILHA et al.

intervention is an important tool for managing gestational diabetes mellitus, and potentially benefits the mother's and fetal health. Nutritional status assessment of the mother should include anthropometric, dietary, biochemical, clinical and functional indicators. In this way, dietary assessment should be thorough, paying attention to the number and kind of meals, and the food groups present. The distribution of macronutrients in relation to the total energy intake per day should be $45-65 \%$ carbohydrates, $15-20 \%$ proteins and $20-35 \%$ lipids. The allowed sweeteners are acesulfame K, aspartame, neotame, saccharin and sucralose. Physical activity should also be included in the strategy to treat diabetes mellitus but the impact of physical activity on neonatal complications still requires minute testing. In addition, some studies have associated nutritional counseling skill with greater adherence to dietary recommendations. Several measures should be taken to ensure successful management of gestational diabetes mellitus, including participation of an inter and multidisciplinary team, early prenatal care with opportune nutritional care, and guaranteed quality care during pregnancy.

Indexing terms: Prenatal care. Diabetes, gestational. Pregnant. Nutrition.

\section{N T R O D U ÇÃ O}

O Diabetes Mellitus Gestacional (DMG) é definido pela diminuição da tolerância à glicose, que se inicia ou é reconhecida pela primeira vez na gestação, podendo ou não persistir após o parto. Essa definição pode incluir pacientes com características clínicas de Diabetes Mellitus (DM) tipo 1 ou tipo 2 e casos de tolerância à glicose diminuída, cujo diagnóstico tenha sido feito somente na gestação atual ${ }^{1,2}$.

A prevalência de DMG situa-se ao redor de $1 \%$ e $14 \%$, dependendo da população estudada, da etnia, da área geográfica, da frequência do rastreamento e dos critérios diagnósticos utilizados $^{1,2}$. O DMG gestacional representa cerca de $90 \%$ de todas as formas de manifestação do diabetes na gestação, ocorrendo o agravamento da intolerância à glicose com mais frequência no terceiro trimestre de gestação".

No Brasil, a prevalência de DMG em muIheres com mais de 20 anos, atendidas em serviços de pré-natal do Sistema Único de Saúde (SUS), é de $7,6 \%$, sendo que $94,0 \%$ dos casos apresentam apenas tolerância diminuída à glicose e $6,0 \%$ hiperglicemia semelhantemente ao nível de diabetes fora da gravidez ${ }^{3}$.

Dentre os fatores de risco destacam-se: idade superior a 25 anos, história pessoal, antecedente familiar de diabetes (parentes de $1^{\circ} \mathrm{grau}$ ), antecedente obstétrico (macrossomia, polidrâmnio, morte fetal ou neonatal de causa desconhe- cida, malformações congênitas e restrição do crescimento fetal), exame obstétrico atual evidenciando: ganho de peso excessivo, altura uterina maior do que a esperada para idade gestacional, crescimento fetal excessivo e polidrâmnio. Também são considerados fatores de risco baixa estatura $(<1,50 \mathrm{~m})$, disposição central excessiva de gordura corporal e doença hipertensiva ${ }^{1,2}$.

Montenegro et al. ${ }^{4}$, investigando 143 pacientes com DMG e seus conceptos, identificaram as principais complicações materno-fetais associadas e suas respectivas frequências: infecção do trato urinário (11,9\%), hipertensão arterial sistêmica $(11,2 \%)$, doença hipertensiva específica da gestação $(9,8 \%)$, abortamento $(0,7 \%)$, hipoglicemia neonatal $(48,6 \%)$, prematuridade $(19 \%)$, macrossomia $(24,6 \%)$, icterícia neonatal $(25,4 \%)$, e malformações $(1,4 \%)$.

Segundo Farrell et al. ${ }^{5}$, a incidência de anomalias congênitas é maior entre mulheres com diabetes pré-gestacional (5\%-10\%) do que gestacional $(1,4 \%)$, confirmando relatos de trabalhos anteriores. $\mathrm{O}$ aumento dos riscos de malformações em fetos de mulheres com DMG está associado à adoção da terapia insulínica.

Nucci et al. ${ }^{6}$, estudando a associação entre o estado antropométrico materno pré-gestacional e o risco de intercorrências gestacionais em gestantes atendidas pelo SUS, identificaram que a obesidade está associada a condições clínicas, como DMG, corroborando dados da literatura que relacionam o aumento da incidência de DMG em 
mulheres obesas. Tal associação é atribuída à resistência insulínica, mais frequente entre as obesas, combinada com deficiente secreção deste hormônio. Outro estudo verificou que a prevalência e o risco relativo de DMG foram de $10,6 \%$ e de 6,57 em obesas, respectivamente 7 .

É consensual na literatura a importância do cuidado nutricional para o adequado controle glicêmico em gestantes com DMG, o que contribui para um bom resultado obstétrico. Em pacientes com DM, inclusive DMG, está comprovado que terapia nutricional e exercício físico devem ser incorporados ao tratamento desta condição clínica ${ }^{1,2}$.

Durante a gravidez, o estado nutricional adequado, resultante da associação alimentação equilibrada e ganho de peso dentro das faixas de normalidade, pode contribuir para a diminuição da morbimortalidade materno-infantil. Assim, considerando-se que o Diabetes Mellitus Gestacional representa uma das situações de alto risco gestacional, com importante interface ao cuidado nutricional, este artigo tem como objetivo revisar na literatura atualidades sobre a terapia nutricional diante desta condição clínica, o que pode auxiliar no controle adequado da gestação e reduzir os riscos perinatais relacionados ao DMG.

\section{MÉ T O D O S}

Trata-se de uma revisão do conhecimento disponível na literatura científica sobre a terapia nutricional no DMG, sem restrição de data e com fontes primárias indexadas nas bases de dados SciELO, PubMed, Medline. Foram também incluídos dados de manuais de comitês de saúde nacionais e internacionais. Adotou-se, para consulta às bases de dados, os seguintes descritores: pregnancy, nutrition, diabetes and gestational diabetes, prenatal care.

\section{RASTRE A MENTO E DIAG NÓSTICO}

O rastreamento universal da abordagem inicial da assistência pré-natal preconizado pelo Ministério da Saúde (MS) ${ }^{8}$ justifica-se não somente àquelas gestantes com fatores de risco associados. Dessa forma, o rastreamento do DMG inicia-se na primeira consulta pré-natal com a solicitação da glicemia de jejum. Nos casos de gestantes com valores superiores a $85 \mathrm{mg} / \mathrm{dL}$ de glicemia de jejum o rastreamento é considerado positivo ${ }^{2,8}$. O diagnóstico é estabelecido com a glicemia de jejum e do teste de tolerância à glicose.

No Quadro 1 é apresentada a referência para interpretação dos resultados de glicemia de jejum e do teste de tolerância à glicose para diagnóstico do Diabetes Mellitus Gestacional.

Nos casos de confirmação do DMG a glicemia materna deverá ser reavaliada partir de seis semanas após o parto, empregando-se a glicemia de jejum ou o teste oral de tolerância com $75 \mathrm{~g}$ de glicose, considerando-se valores de normalidade inferiores a $110 \mathrm{mg} / \mathrm{dL}$ e $140 \mathrm{mg} / \mathrm{dL}$, respectivamente ${ }^{2}$.

Quadro 1. Interpretação dos resultados de glicemia de jejum e do teste de tolerância à glicose para diagnóstico do Diabetes Mellitus Gestacional.

\begin{tabular}{lcc}
\hline Classificação & Glicemia de jejum $(\mathrm{mg} / \mathrm{dL})$ & Glicemia $2 \mathrm{~h}$ após TTG- 75g (mg/dL) \\
\hline Normal & $<110$ & $<140$ \\
\hline Regulação glicêmica alterada & & \\
- Glicemia de jejum & $110-125$ & $140-199$ \\
- Tolerância à glicose diminuída & 110 & \\
\hline
\end{tabular}

Fonte: Brasili

TTG- 75g: teste de tolerância à glicose com $75 \mathrm{~g}$ de glicose. 


\section{FISIOPATOLOGIA DO DIABETES NA GESTAÇÃO}

A gravidez altera hábitos alimentares, padrões de atividade física, estado emocional, sensibilidade à insulina e produção de hormônios. Essas mudanças alteram o metabolismo de carboidrato e as necessidades de insulina. Na gestante não-diabética, os hormônios placentários e ovarianos estimulam maior secreção de insulina, porém ocorre alteração na sensibilidade celular devido às alterações nos receptores de insulina ${ }^{9}$.

No início da gestação os níveis elevados de estrogênio e progesterona determinam hiperplasia das células $\beta$-pancreáticas, aumentando a resposta da insulina a uma carga de glicose. $\mathrm{O}$ objetivo deste aumento é facilitar a lipogênese e a gliconeogênese, favorecendo maiores depósitos de glicogênio, triglicerídeos e proteínas, condição de anabolismo facilitada ${ }^{10,11}$.

Na segunda metade da gestação, o hormônio lactogênio placentário (HPL), um polipeptídeo de estrutura e função semelhantes às do hormônio do crescimento, acarreta maior secreção de insulina, apesar de diminuir sua sensibilidade no âmbito celular, estimula a lipólise e a gliconeogênese, sendo classificado como um hormônio catabólico. Desse modo, no segundo trimestre começam a atuar os fatores hiperglicemiantes e contrainsulínicos, elevando-se rapidamente a glicemia e, como conseqüência, as necessidades de insulina. Neste período modifica-se a tendência materna de anabolismo para catabolismo, visando a atender as crescentes necessidades fetais ${ }^{10}$.

No terceiro trimestre acontece a maior alteração da glicemia, devido à elevada mobilização de glicogênio. Assim, gestantes com reservas pancreáticas limitadas de insulina desenvolvem o quadro de diabetes. Neste período da gestação continuam crescentes as necessidades de insulina até as últimas semanas, quando se inicia a senescência placentária e se reduz a produção de fatores hiperglicemiantes ${ }^{10}$.
O DMG resulta da incapacidade de as mulheres aumentarem a secreção de insulina, na maioria das vezes determinada por um defeito funcional, não imunológico nas células $\beta$-pancreáticas, que prejudica a capacidade de compensar a resistência insulínica da gravidez ${ }^{12}$. Alguns autores sugerem como prováveis mecanismos para esta descompensação a mutação do gene da glicoquinase ${ }^{13}$, a resistência insulínica associada à idade, à obesidade, ao sedentarismo, ao estilo de vida e à história familiar ${ }^{14}$ e o defeito nos receptores de glicose nos adipócitos ${ }^{15}$.

Vários fatores contribuem para tais afirmações, dentre eles: a degradação placentária da insulina, o aumento do nível sérico dos ácidos graxos, a elevação dos níveis séricos dos glicocorticóides, o aumento dos teores de estrógeno e progesterona e a produção crescente do $\mathrm{HPL}^{16}$.

Os níveis de estrógeno e progesterona produzidos pela placenta, aumentam na gestação e são responsáveis, em parte, pelas alterações do metabolismo glicídico materno. Desse modo, o estrógeno age como antagônico à insulina e a progesterona também afeta o metabolismo da glicose, agindo como antagonista da insulina, diminuindo sua eficácia nos tecidos periféricos ${ }^{16}$. O cortisol está aumentado no final da gestação, e atua reduzindo a sensibilidade tecidual à insulina. Eleva os níveis glicêmicos estimulando a gliconeogênese dos aminoácidos e antagonizando a ação da insulina no músculo e no tecido adiposo ${ }^{10}$.

Na maioria dos casos de DMG, com a redução dos níveis de hormônios contrainsulínicos no período pós-parto, as necessidades maternas de insulina e os níveis de glicemia retornam aos valores pré-gestacionais'. Por outro lado, mulheres que apresentam DMG têm elevado risco de desenvolver Diabetes tipo 2 posteriormente ${ }^{14,17,18}$.

Lee et al. ${ }^{19}$, em estudo de coorte retrospectivo com 5470 mulheres acometidas previamente por DMG e 783 controles, encontraram risco acumulado de 9,6 (5,9-16,7) vezes maior entre as mulheres com história de DMG. Em recente revisão sistemática da literatura, Kim et al. ${ }^{20}$ observaram que a recorrência de DMG variou entre $30 \%$ e $84 \%$ dos estudos avaliados. 
O estado nutricional da mulher, antes e durante a gestação, é crítico para um resultado obstétrico favorável. Na avaliação do estado nutricional materno devem ser empregados os indicadores antropométricos e outros indicadores classificados como dietéticos, bioquímicos, clínicos e funcional ${ }^{21}$. Os antropométricos são de baixo custo, têm caráter pouco invasivo e fácil aplicabilidade, sendo essenciais para identificar mulheres em risco de complicações gestacionais, dentre elas o DMG ${ }^{22,23}$.

É importante destacar que os princípios da avaliação nutricional são aplicáveis para todos os casos de diabetes, tanto gestacional quanto prévia.

A avaliação antropométrica deve ser realizada, inicialmente, pela determinação do Índice de Massa Corporal (IMC) pré-gestacional, que permite classificar o estado nutricional da gestante antes da concepção e identificar o ganho de peso adequado, segundo cada categoria de estado antropométrico pré-gestacional (Quadro 2).

A avaliação dietética deve ser detalhada, com atenção para o fracionamento e a composição das refeições, e para os grupos de alimentos presentes. O método de inquérito dietético mais empregado é o de frequência de consumo se- miquantitativo, constituído de lista de alimentos divididos por grupos de alimentos, categorias de frequência de consumo e quantidade usual de consumo para cada alimento, fornecendo uma estimativa da alimentação habitual do indivíduo, quantitativa e qualitativamente. Deve-se investigar também a utilização dos edulcorantes presentes nos adoçantes de mesa e nos produtos diet ou light, visando a identificar o uso de edulcorantes contraindicados no período gestacional ${ }^{10,21}$.

Na avaliação clínica devem-se investigar os sinais e sintomas digestivos mais comuns durante a gestação e que, na dependência de severidade do quadro, podem interferir na ingestão dos alimentos e, portanto, no ganho de peso gestacional. Sugere-se também o rastreamento de outras intercorrências gestacionais, pela avaliação bioquímica de: hemoglobina e hematócrito, perfil lipídico, proteinúria, função hepática e renal; de dados clínicos, como pressão arterial, presença de edema e sinais sugestivos de carências nutricionais específicas, tais como, cegueira noturna, bócio, palidez conjuntival, dentre outros ${ }^{21,30}$.

Na avaliação funcional da Deficiência de Vitamina A (DVA) pode-se adotar a entrevista padronizada (Quadro 3), que identifica a Cegueira

Quadro 2. Classificação de estado nutricional segundo IMC pré-gestacional, ganho de peso no primeiro trimestre, semanal, total e mínimo para gestantes adultas.

\begin{tabular}{|c|c|c|c|c|c|}
\hline $\mathrm{IMC}^{1}\left(\mathrm{~kg} / \mathrm{m}^{2}\right)$ & $\begin{array}{c}\text { Estado } \\
\text { nutricional }\end{array}$ & $\begin{array}{c}\text { Ganho de peso } \\
(\mathrm{kg}) \text { no } 1^{\circ} \text { trimestre } \\
\left(\mathrm{IG}<14^{\mathrm{a}} \mathrm{s}\right)^{2}\end{array}$ & $\begin{array}{l}\text { Ganho de peso }(\mathrm{kg} / \\
\text { sem) no } 2^{\circ} \text { e } 3^{\circ} \\
\text { trimestre }\left(I G \geq 14^{\mathrm{a}} \mathrm{s}\right)\end{array}$ & $\begin{array}{c}\text { Ganho de peso } \\
(\mathrm{kg}) \text { total }\end{array}$ & $\begin{array}{c}\text { Ganho de peso }(\mathrm{kg}) \\
\text { mínimo no } 2^{\circ} \mathrm{e} \\
3^{\circ} \text { trimestre }^{3}\end{array}$ \\
\hline$<8,5$ & Baixo peso & 2,3 & 0,5 & $12,5-18,0$ & Não determinado \\
\hline $18,5-24,9$ & Normal & 1,6 & 0,4 & $11,5-16,0$ & $1,0 \mathrm{~kg} / \mathrm{mês}$ ou $0,25 \mathrm{~kg} / \mathrm{semana}$ \\
\hline $25,0-29,9$ & Sobrepeso & 0,9 & 0,3 & $7,0-11,5$ & Não determinado \\
\hline$\geq 30,0$ & Obesidade & - & $0,3^{4}$ & 7,0 & $0,5 \mathrm{~kg} / \mathrm{mês}$ ou $0,125 \mathrm{~kg} / \mathrm{semana}$ \\
\hline
\end{tabular}

Fonte: Adaptado de Brasil ${ }^{8}$, World Health Organization ${ }^{23}$, Institute of Medicine ${ }^{24-25}$, Padilha ${ }^{26}$, Saunders et a ${ }^{27}$.

IMC: Índice de Massa Corporal.

1 Pontos de corte para classificação do estado nutricional antropométrico pré-gestacional de gestantes adultas, que permite a identificação das mulheres em risco de resultado obstétrico indesejável (baixo peso ao nascer) ${ }^{26}$. Para as gestantes adolescentes, devem-se adotar os pontos de corte de IMC próprios para a idade materna ${ }^{27}$.

2 Para as gestantes que iniciam o cuidado nutricional no primeiro trimestre, deve-se programar o ganho de peso gestacional total para o período (até a o final da $13^{\mathrm{a}}$ semana gestacional), conforme a categoria de IMC pré-gestacional.

${ }^{3}$ Na prática clínica ocorrem situações em que a gestante já atingiu o ganho de peso final sugerido no Quadro 1. Nestes casos, pode-se adotar a definição de ganho mínimo ou modesto, que é de 1,0kg/mês para as mulheres com IMC pré-gestacional normal e de 0,5kg/mês para as com obesidade pré-gestacional23.

${ }^{4}$ É proposto o ganho de peso mínimo de $0,3 \mathrm{~kg} / \mathrm{semana}$ no $2^{\circ}$ e no $3^{\circ}$ trimestres gestacionais, que está associado ao melhor resultado obstétrico ${ }^{28}$. 
Quadro 3. Entrevista padronizada para diagnosticar cegueira noturna.

Dificuldade para enxergar durante o dia?

Dificuldade para enxergar com pouca luz ou à noite?

Tem cegueira noturna?

Fonte: Saunders et al. ${ }^{31}$

Noturna (XN) gestacional. Tal entrevista foi validada, segundo o indicador bioquímico (níveis séricos de retinol) em puérperas atendidas em Maternidade Pública do Rio de Janeiro ${ }^{31}$. São considerados casos de $\mathrm{XN}$ quando a resposta para a pergunta 1 for não é a resposta para a pergunta 2 e/ou 3 for sim. Caso a entrevistada gestante apresente algum problema ocular corrigido por óculos ou por lente de contato, deve ser questionada a capacidade de visão com o uso destes ${ }^{31}$. O termo cegueira noturna trata-se de uma alteração do padrão habitual de visão com pouca luz ou à noite ou dificuldade de adaptação da visão na mudança de um ambiente claro para um escuro, adotando-se como referência o padrão de visão noturna no período pré-gestacional|31,32.

A XN geralmente é diagnosticada no segundo ou no terceiro trimestre gestacional, também havendo maior risco de $\mathrm{XN}$ após o $3^{\circ}$ mês de amamentação. A presença de $\mathrm{XN}$ gestacional é considerada atualmente preditora de risco, pois constatou-se associação entre a presença da $\mathrm{XN}$ e anemia, síndromes hipertensivas da gestação, infecções e infecção puerperal e maior risco de mortalidade materna por infecção respiratória e por outras infecções ${ }^{32,33}$.

Vale ressaltar que a XN gestacional acomete mulheres independentemente do seu estado antropométrico pré-gestacional ou gestacional e das suas condições socioeconômicas, podendo estar associada a um menor número de consultas da assistência pré-natal e à história de aborto. Assim, sugere-se que a investigação da XN deve ser feita no universo de gestantes atendidas nos serviços de pré-natal ${ }^{34}$. Em indivíduos portadores de DM tipo 1 é descrita a dificuldade na biocon- versão da pró-vitamina $A$ em vitamina $A$ ativa. Portanto, na orientação nutricional deve-se estimular o consumo dos alimentos fonte de origem animal da vitamina ${ }^{10}$.

\section{TERAPIA NUTRICIONAL}

A atenção integrada à gestante com diagnóstico de DMG é de fundamental importância para diminuir as possíveis complicações advindas desta condição clínica. Devem ser oferecidos orientação nutricional, controle metabólico, insulinoterapia, prática de atividade física, assistência pré-natal e avaliação do bem-estar fetal' ${ }^{1}$.

O tratamento inicial consiste de controle dietético adequado, cujos objetivos são prover adequada nutrição materno-fetal e ganho ponderal recomendado, assim como atingir e manter o controle metabólico adequado. Este inclui níveis de glicemia na faixa de normalidade, isto é, glicemia de jejum inferior a $95 \mathrm{mg} / \mathrm{dL}(5,3 \mathrm{mmol} / \mathrm{L})$ e a pós-prandial (2h) inferior a 120 mg/dL (6,8 mmol/L). Deve-se ainda prover energia e nutrientes suficientes para um bom resultado obstétrico, manter perfil lipídico e pressão arterial controlados e prevenir o desenvolvimento de DM tipo 2 no pós-parto1,35,36.

Na dieta deve-se priorizar uma distribuição harmônica dos macronutrientes, podendo variar de acordo com as considerações específicas para cada macronutriente. Recomenda-se que os carboidratos devam perfazer um total de $45-65 \%$ do Valor Energético Total (VET), as proteínas 15-20\% (com um adicional diário de $10 \mathrm{~g}^{35,36}$ ou 1,1 $/ \mathrm{kg}$ de peso pré-gestacional/dia, com a ingestão total recomendada de $71 \mathrm{~g} / \mathrm{dia}$ ) e os lipídios de $20 \%-35 \%{ }^{35-37}$.

A American Dietetic Association (ADA $)^{1,36}$ faz algumas considerações a respeito da distribuição dos macronutrientes. Quanto aos carboidratos destaca que dietas com um quantitativo inferior a $130 \mathrm{~g}$ não devem ser prescritas com a finalidade de melhor controle glicêmico, podendo trazer efeitos deletérios. Considerando-se as proteínas, o referido comitê destaca que a Ingestão Dietética 
de Referência (IDR) ${ }^{37}$ considera como distribuição aceitável um total de 10\%-35\% do VET, priorizando-se as fontes protéicas de alto valor biológico. Considera ainda que para pacientes com diabetes deva ser recomendado menos de $7 \%$ do total de lipídeos na forma de gordura saturada e que a ingestão de colesterol diária seja inferior a 200mg/ dia. Entretanto, não faz menção aos casos de $\mathrm{DMG}^{1,36}$.

A necessidade de vitaminas e minerais e o aporte de fibras (20-35g/dia ou 14g/1000kcal) é semelhante ao recomendado para gestantes não acometidas pelo diabetes ${ }^{35,36}$.

A recomendação de energia proposta para gestantes com DMG é ainda contraditória, porém é consenso que a energia recomendada deve prover o ganho de peso adequado e que dietas com restrições severas de energia não são aconselhadas, pois podem determinar a cetonúria. Por outro lado, alguns autores relatam que uma restrição moderada (até 30\% das necessidades estimadas) de energia, em média $25 \mathrm{kcal} / \mathrm{kg}$, em gestantes obesas $\left(\mathrm{IMC}>30 \mathrm{~kg} / \mathrm{m}^{2}\right)$, mantém a euglicemia, sem elevação plasmática de ácidos graxos livres ou cetonúria $35,36,38$.

Em relação ao efeito do índice glicêmico dos carboidratos, afirma-se que a quantidade de carboidrato por refeição é mais importante do que a fonte ou o tipo de deste $2,35,36$. Embora dietas com baixo índice glicêmico possam reduzir a glicemia pós-prandial e o perfil lipídico, a capacidade dos indivíduos para manter essas dietas a longo prazo não é definida claramente na literatura. Nesse sentido, o uso de alimentos de baixo índice glicêmico, como estratégia primária no plano alimentar, não encontra evidência suficiente $2,35,36$.

Ao longo dos últimos anos, alguns estudiosos têm enfatizado que, dietas com consumo de carboidratos controlados e cuidadosa distribuição destes ao longo do dia são capazes de controlar a glicemia, reduzir a incidência de macrossomia e a necessidade de insulina ${ }^{35,39}$. Porém, a quantidade e a distribuição de carboidratos para mulheres acometidas pelo DMG ainda é questionada, sendo recomendável que a ingestão total de carboidratos seja individualizada, de acordo com a capacidade da gestante em tolerá-los, suas necessidades e hábitos alimentares $^{40}$.

Contrariamente, Zhang et al. ${ }^{41}$, investigando a relação do consumo pré-gestacional de fibras (frutas, vegetais e cereais) e o índice glicêmico concluíram que, a cada $10 \mathrm{~g} / \mathrm{dia}$ do consumo total houve redução de $26 \%$ do risco de DMG. 0 mesmo estudo indicou que o índice glicêmico esteve positivamente associado ao DMG, concluindo que a dieta pré-gestacional parece estar associada ao risco de DMG, em especial as dietas com alto índice glicêmico e baixo teor de fibras.

O plano alimentar deve ser fracionado com menor volume, horário rígido, e intervalos regulares, sendo propostas em torno de 5-6 refeições por dia.

Os educorantes liberados para utilização pela população em geral, inclusive para gestantes, são acesulfame K, aspartame, neotame, sacarina e sucralose. Recomenda-se o uso moderado de educorantes uma vez que não existem estudos conclusivos realizados com humanos sobre sua seguranção na gestação (Quadro 4) e evitar o consumo de alimentos diet e adoçantes à base de frutose, sorbitol, stévia e ciclamato ${ }^{36,42}$. O consumo de frutose, sob a forma de adoçante, deve ser desestimulado, por aumentar os níveis de lipídeos plamáticos. Porém, não se recomenda que indivíduos evitem a frutose naturalmente presente nos alimentos ${ }^{36,42}$

A partir do relatório da ADA recomenda-se como mais uma ferramenta nutricional, o mé-

Quadro 4. Educorantes permitidos para gestantes e ingestão diária máxima para adultos.

\begin{tabular}{lcc}
\hline Edulcorante & $\begin{array}{c}\text { Valor energético } \\
(\mathrm{kcal} / \mathrm{g})\end{array}$ & $\begin{array}{c}\text { Ingestão diária máxima } \\
(\mathrm{mg} / \mathrm{kg} / \mathrm{dia})\end{array}$ \\
\hline Acesulfame $-\mathrm{K}^{\text {Aspartame }}$ & 0 & 15 \\
Neotame $_{\text {Sacarina }}$ & 4 & 40 \\
Sucralose & 0 & 2 \\
\hline
\end{tabular}

a Devido ao seu alto poder adoçante, a quantidade utilizada é pequena e a energia derivada é insignificante.

Fonte: American Dietetic Association ${ }^{42}$ 
todo de contagem de carboidratos, que visa ao controle glicêmico em função das menores variações das glicemias pós-prandiais. Atualmente, vários grupos utilizam a contagem de carboidratos de forma sistemática, o que é recomedado pela Sociedade Brasileira de Diabetes (SBD ${ }^{43}$.

Os carboidratos provenientes dos alimentos integrais, das frutas, dos vegetais e dos produtos lácteos com menor teor de gordura devem ser priorizados. A sacarose não necessita ser restrita, podendo ser incluída no total de carboidratos da dieta, respeitando o contexto da alimentação saudável ${ }^{36}$.

A adesão ao plano alimentar saudável, atendendo aos princípios de quantidade, qualidade, padrão das refeições e adequação do ganho de peso, é fundamental para o bom controle glicêmico, contribuindo para a incorporação de adequado estilo de vida, mesmo após o término da gestação ${ }^{35,36}$.

O consumo de bebidas alcoólicas deve ser desencorajado no período gestacional, não somente pelo risco de hipoglicemia materna, mas também pelo risco aumentado de síndrome alcoólica fetal, resultante do uso crônico do álcool durante a gravidez, cujas consequências variam desde sequelas não aparentes até a restrição do crescimento fetal intrauterino e lactente, com danos graves ${ }^{35,36}$. Além disso, as bebidas alcoólicas fornecem calorias que são mais difíceis de ser metabolizadas pela mãe e pelo feto, e desprovidas de proteínas, minerais e vitaminas. Como resultado, a absorção, o metabolismo e o uso dos nutrientes ficam prejudicados ${ }^{9}$.

A habilidade de aconselhamento nutricional é muito útil na abordagem aos casos de DMG, com o intuito de melhorar a adesão ao cuidado nutricional, contribuindo para um resultado obstétrico favorável ${ }^{30}$.

Crowther et al. ${ }^{44}$, avaliando o impacto da intervenção nutricional nos resultados perinatais, observaram menor taxa de complicações perinatais em mulheres que receberam a intervenção nutricional padronizada por nutricionistas especializados, quando comparadas àquelas que receberam acompanhamento tradicionalmente aplicado nas rotinas, considerando-se o peso pré-gestacional, o ganho ponderal durante a gestação, o nível de atividade física e os hábitos alimentares.

Em estudo de validação de um guia para o controle nutricional de mulheres com DMG, Reader et al. ${ }^{45}$ concluem que o cuidado nutricional específico, com a utilização de um protocolo, tende a melhorar o resultado obstétrico.

A atividade física deve fazer parte da estratégia de tratamento do DMG, embora o impacto do exercício nas complicações neonatais ainda mereça ser rigorosamente testado. Recomenda-se que mulheres sem complicações obstétricas e clínicas sejam encorajadas a iniciar ou continuar um programa de exercício moderado, como parte do tratamento ${ }^{35,36}$. De acordo com a SBD², pacientes sedentárias podem ser orientadas a iniciar um programa de caminhadas regulares e/ou de outros exercícios de baixo impacto, e as gestantes praticantes de exercícios regulares previamente à gestação podem manter atividades físicas habituais, evitando exercícios de alto impacto ou que predisponham à perda de equilíbrio.

Quando o tratamento com dieta e exercício físico não consegue o controle glicêmico desejado, inicia-se a administração concomitante de insulina. A necessidade e a resposta à insulina exógena são individuais, exigindo doses e esquemas diferentes para cada paciente.

No pós-parto medidas de incentivo ao aleitamento materno devem ser adotadas, e, caso ocorra hiperglicemia, o uso de insulina pode ser indicado. Ademais, deve-se evitar a recomendação de dietas hipoenergéticas durante a amamentação 2,35,36. Evidências científicas também apontam o aleitamento materno como uma medida de prevenção contra o DM tipo 2 entre filhos de mães com DMG. Entretanto, especula-se a sua contribuição para perda ponderal pós-parto e na regulação da necessidade de insulina ${ }^{46,47}$.

O Quadro 5 apresenta, resumidamente, as recomendações nutricionais de macro e micronutrientes para gestantes com DMG. 
Quadro 5. Recomendações nutricionais de macro e micronutrientes para gestantes com diabetes.

\begin{tabular}{|c|c|c|}
\hline Macronutrientes & Recomendação & Observações \\
\hline Carboidratos & 45 a $65 \%$ do VET & Fibras solúveis e insolúveis devem ser de 20 a 35g/dia, ou 14g/1000kcal \\
\hline Proteínas & 15 a $20 \%$ do VET & $\begin{array}{l}\text { Adicional diário para o período gestacional de } 10 \mathrm{~g} \text { ou } 1 \mathrm{~g} / \mathrm{kg} \text { de peso } \\
\text { pré-gestacional/dia, com a ingestão total recomendada de } 71 \mathrm{~g} / \mathrm{dia}\end{array}$ \\
\hline \multirow[t]{3}{*}{ Lipídios } & $20-35 \%$ do VET & $<7 \%$ do VET de gordura saturada \\
\hline & & Colesterol:<200mg/dia. \\
\hline & & $\begin{array}{l}\text { Estimular o consumo de } 2-3 \text { porções de peixe por semana para o fornecimen- } \\
\text { to de ácidos graxos n-3 }\end{array}$ \\
\hline
\end{tabular}

\begin{tabular}{|c|c|}
\hline \multicolumn{2}{|r|}{ Vitaminas e minerais } \\
\hline Sódio & Não deve ser restrito, à exceção dos casos de hipertensão arterial grave \\
\hline \multirow{2}{*}{ Ferro } & $\begin{array}{l}\text { - Hemoglobina }>11 \mathrm{~g} / \mathrm{dL} \text { : ausência de anemia; suplementação de } 60 \mathrm{mg} / \mathrm{dia} \text { de ferro elementar, a } \\
\text { partir da } 20^{\mathrm{a}} \text { semana, associada a } 5 \mathrm{mg} / \mathrm{dia} \text { de ácido fólico. Recomenda-se ingestão uma hora } \\
\text { antes das refeições }\end{array}$ \\
\hline & $\begin{array}{l}\text { - Hemoglobina }<11 \mathrm{~g} / \mathrm{dL} \text { e }>8 \mathrm{~g} / \mathrm{dL} \text { : diagnóstico de anemia leve a moderada. Suplementação com } \\
\text { sulfato ferroso ( } 120 \text { a } 240 \mathrm{mg} \text { de ferro elementar/dia), de } 2 \text { a } 4 \text { drágeas de sulfato ferroso, via oral/ } \\
\text { dia, uma hora antes das principais refeições. }\end{array}$ \\
\hline $\begin{array}{l}\text { Vitaminas C e E, Selênio, } \\
\text { Beta-caroteno e outros } \\
\text { carotenóides }\end{array}$ & $\begin{array}{l}\text { Estimular a ingestão de alimento fontes, em função da ação antioxidante para prevenir o stress } \\
\text { oxidativo, porém não existe um consenso sobre a recomendação de suplementação em indivíduos } \\
\text { com diabetes. }\end{array}$ \\
\hline $\begin{array}{l}\text { Potássio, Magnésio, Zinco e } \\
\text { Cromo }\end{array}$ & $\begin{array}{l}\text { A dieta deve ser adequada nesses nutrientes, pois suas deficiências podem agravar a hiperglicemia, } \\
\text { porém os estudos ainda são pouco conclusivos quando relacionados à suplementação. }\end{array}$ \\
\hline
\end{tabular}

Fontes: Brasil'; Saunders \& Padilha ${ }^{10}$; American Diabetes Association ${ }^{35,36}$.

VET: valor energético total.

\section{O N CLUS Ã O}

A intervenção nutricional é uma importante aliada no controle do Diabetes Mellitus Gestacional, trazendo benefícios à saúde materno-fetal. É unânime o reconhecimento de que as necessidades nutricionais durante o período gestacional e a lactação são similares às das mulheres sem diabetes. Assim, a terapia nutricional para DMG deve estar focada em escolhas alimentares que garantam um apropriado ganho de peso, controle glicêmico e ausência de corpos cetônicos.

Para o sucesso do controle do DMG são necessários: a participação da equipe inter e multidisciplinar, o cuidado pré-natal precoce, com assistência nutricional oportuna e a garantia da assistência de qualidade ao longo da gestação, permitindo a intervenção a partir de orientação nutricional individualizada e de qualidade, o que reflete na adequação dos ajustes fisiológicos gestacionais, tornando o meio favorável ao binômio mãe-filho.

\section{A GRADECIMENTOS}

Aos órgãos de fomento de pesquisa, Fundação Carlos Chagas Filho de Amparo à Pesquisa do Estado do Rio de Janeiro e a Conselho Nacional de Desenvolvimento Científico e Tecnológico. 
104 | P.C. PADILHA et al.

\section{COLABORADORES}

P.C. PADILHA, E. ACCIOLY e C. SAUNDERS participaram na concepção e no planejamento do estudo, na coleta de dados, na análise crítica dos resultados, na elaboração e na revisão da versão final do manuscrito. A.B. SENA, J.L. NOGUEIRA, R. P.S. ARAÚJO e P.D. ALVES participaram na coleta de dados, na análise dos resultados e na elaboração de versões preliminares do manuscrito.

\section{REFERÊ NCIAS}

1. American Diabetes Association. Standards of medical care in diabetes-2007. Diabetes Care. 2007; 30 (Suppl 1):s4-s41. doi 10.2337/dc07-S004.

2. Milech A, Forti AC, Golbert A, Ramalho AC, Lerário $A C$, Pires AC, et al. Tratamento e acompanhamento do diabetes mellitus: Diretrizes da Sociedade Brasileira de Diabetes, 2006 [Acesso 2010 jan 25]. Disponível em: <http://www.diabetes.org.br/ educacao/docs/diretrizes.pdf>.

3. Brasil. Ministério da Saúde. Gestação de alto risco: manual técnico. Brasília: Ministério da Saúde; 2000.

4. Montenegro RM, Paccola GMFG, Farias MC. Montenegro APDR, Jorge SM. Evolução maternofetal de gestantes diabéticas seguidas no $\mathrm{HC}$ / FMRP/USP no período de 1992-1999. Arq Bras Endocrino Metabol. 2001; 45(5):467-74. doi: 10.1590/S0004-27302001000500010.

5. Farrel T, Neale L, Cundy T. Congenital anomalies in the offspring of women with type 1, type 2 and gestational diabetes. Diabet Med. 2001; 19(4): 322-6. doi 10.1046/j.1464-5491.2002.00700.

6. Nucci LB, Schimidt M I, Duncan BB, Fuchs SC, Fleck ET, Britto MMS. Nutritional status of pregnant women: prevalence and associated pregnancy outcames. Rev Saúde Pública. 2001; 35(6): 502-7. doi 10.1590/S0034-89102001000600002.

7. Abrams BF, Parker J. Overweight and pregnancy complications. Int J Obes. 1988; 12 (4):293-303.

8. Brasil. Ministério da Saúde. Pré-natal e Puerpério: atenção qualificada e humanizada. Manual técnico. Brasília, 2006 [acesso 2008 jul 29]. Disponível em: <www.saude.gov.br>.

9. McGanity WJ, Dawson EB, Hook, JMV. Nutrição materna. In: Shils ME. Tratado de nutrição moderna na saúde e na doença. Rio de Janeiro: Manole; 2003. p.869-98.

10. Saunders C, Padilha PC. Diabetes na gestação. In: Accioly E, Saunders C, Lacerda E. Nutrição em obste- trícia e pediatria. Rio de Janeiro: Cultura Médica; 2009. p.191-207.

11. Lefebvre $P$, Renard $E$, BrinDMGer J. Diabète et grossesse. Cah Nutr Diét. 1995; 30(5):331-6.

12. Burrow F. Complicações clínicas durante a gravidez. 4a.ed. São Paulo: Roca; 1996. p.189-95.

13. Stoffel M, Bell KL, Blackburn CL, Powell KL, Seo TS, Takeda J, et al. Identification of glucokinase mutations in subjects with gestational diabetes mellitus. Diabetes. 1993; 42(6):937-40.

14. Berdanier CD. Mitochondrial gene expression in diabetes mellitus: effect of nutrition. Nutr Rev. 2001; 59(3 Pt 1):61-70.

15. Garvey WT, Maianu L, Zhu JH, Hancock JA, Golichowski AM. Multiple defects in the adiposity glucose transport system cause celular insulin resistance in gestational diabetes: heterogeneity in the number and a novel abnormality in subcellular localization of GLUT 4 glucose transporters. Diabetes. 1993; 42(12):1773-85.

16. Yamashita $H$, Shao J, Friedman JE. Physiologic and molecular alterations in carbohydrate metabolism during pregnancy and gestational diabetes mellitus. Clin Obstet Gynecol. 2000; 43(1):87-98.

17. Schaefer-Graf UM, Buchanan TA, Xiang AH, Peters RK, Kjos SL. Clinical predictors for a high risk for the development of diabetes mellitus in the early puerperium in women with recent gestational diabetes mellitus. Am J Obstet Gynecol. 2002; 186(4):751-6.

18. Lauenborg J, Hansen $T$, Jensen DM, Vestergaard $H$, Molsted-Pedersen L, Hornnes $P$, et al. Increasing incidence of diabetes after gestational diabetes: a long-term follow-up in a Danish population. Diabetes Care. 2004; 27(5):1194-9.

19. Lee AJ, Hiscock RJ, Wein P, Walker SP, Permezel M. Gestational diabetes mellitus: clinical predictors and long-term risk of developing type 2 diabetes: a retrospective cohort study using survival analysis. Diabetes Care. 2007; 30(4):878-83.

20. Kim C, Berger DK, Chamany S. Recurrence of gestational diabetes: a systematic review. Diabetes Care. 2007; 30:1314-19.

21. Saunders C, Bessa TCAA. Assistência nutricional pré-natal. In: Accioly E, Saunders C, Lacerda EA. Nutrição em obstetrícia e pediatria. Rio de Janeiro: Cultura Médica; 2005. p.119-44.

22. Scotland NE, Haas JS, Brawarsky P, Jackson RA. Body mass index, provider advice, and target gestational weight gain. Obstet Gynecol. 2005; 105(3):633-8.

23. World Health Organization. Promoting optimal fetal development: report of a technical consultation. Geneva: WHO; 2006. 
24. Institute of Medicine. Nutrition during pregnancy. Washington: National Academy Press; 1990.

25. Institute of Medicine. Nutrition during Pregnancy and Lactation: an implementation guide. Washington (DC): National Academy Press; 1992.

26. Padilha PC. Validação de metodologia de avaliação antropométrica de gestantes [dissertação]. Rio de Janeiro: Universidade Federal do Rio de Janeiro; 2006.

27. Saunders C, Accioly E, Costa RSS, Lacerda E. Gestante adolescente. In: Accioly E, Saunders C, Lacerda E. Nutrição em obstetrícia e pediatria. Rio de Janeiro: Cultura Médica; 2005. p.171-88.

28. Siega-Riz AM, Adair LS, Hobel CJ. Maternal underweight status and inadequate rate of weight gain during the third trimester of pregnancy increases the risk of preterm delivery. J Nutr. 1996; 126 (1):146-53.

29. Gutierrez Y, King JC. Nutrition during teenage pregnancy. Pediatr Ann. 1993; 22(2): 99-108.

30. Accioly E, Padilha PC, Saunders C. Nutrição na gestação de alto-risco. Nutr Prof. 2006; 2(8):40-6.

31. Saunders C, Ramalho RA, Lima AP, Gomes M, Campos L, Silva B, et al. Association between gestational night blindness and serum retinol in mother/newborn pairs in the city of Rio de Janeiro, Brazil. Nutrition. 2005; 21(4):456-61. doi:10.1016/ j.nut.2004.07.015.

32. Saunders C, Ramalho A, Padilha PC, Barbosa CC, Leal MC. A investigação da cegueira noturna no grupo materno-infantil: uma revisão histórica. Rev Nutr. 2007; 20(1):95-103. doi: 10.1590/\$1415-52 732007000100010.

33. Christian P. Micronutrients and reproductive health issues: an international perspective. J Nutr. 2002; 133(6):S1969-73.

34. Saunders C, Leal MC, Gomes MM, Campos LFC, Silva BAS, Ramalho RA, et al. Gestational night blindness in women treated at a public maternity hospital in Rio de Janeiro, Brazil. J Health Popul Nutr. 2004; 22(4):348-56.

35. American Diabetes Association. Nutrition recommendations and interventions for diabetes. Gestational diabetes mellitus. Diabetes Care. 2004; 27(Suppl 1):S88-90. doi: 10.2337/diacare. 27.2007.S88.

36. American Diabetes Association. Nutrition recommendations and interventions for diabetes. Diabetes Care. 2008; 30(Suppl 1):561-78. doi: 10.2337/dc08-S061.
37. Institute of Medicine. Dietary reference intakes for energy, carbohydrate. fiber, fat, fatty acids, cholesterol, protein, and amino acids (2002/2005). [cited 2007 Mar 23]. Available from: <www.nap. edu>.

38. Franz MJ, Bantle JP, Beebe CA, Brunzell JD, Chiasson $J-L$, Garg A. Evidence based nutrition principles and recommendations for the treatment and prevention of diabetes and related complications (TechnicalReview). Diabetes Care. 2002; 25 (1): 148-98. doi: 10.2337/diacare.25.1.148.

39. Major CA, Henry MJ, De Veciana M, Morgan MA. The effects of carbohydrate restriction in patients with diet-controlled gestational diabetes. Obstet Gynecol. 1998; 91(4):600-4.

40. Marcason W. What is the appropriate amount and distribution of carbohydrates for a woman diagnosed with gestational diabetes mellitus? J Am Diet Assoc. 2005; 105(10):1673.

41. Zhang C, Liu S, Solomon CG, Hu FB. Dietary fiber intake, dietary glycemic load, and the risk for gestational diabetes mellitus. Diabetes Care. 2006; 29(10):2223-30. doi: 10.2337/dc06-0266.

42. American Dietetic Association. Position of the American Dietetic Association: use of nutritive and Nonnutritive sweeteners. J Am Diet Assoc. 2004; 104(2):255-75. doi:10.1016/j.jada.2003.12.001.

43. Monteiro JBR, Mendonça DRB, Goveia GR, Bruno $L$, Merino M, Sachs A. Manual oficial de contagem de carboidratos. Sociedade Brasileira de Diabetes. Rio de Janeiro; Diagraphic, 2003.

44. Crowther CA, Hiller JE, Moss JR, McPhee AJ, Jeffries WS, Robinson JS. Australian carbohydrate intolerance study in pregnant women (ACHOIS) trial group. Effect of treatment of gestational diabetes mellitus on pregnancy outcomes. $\mathrm{N} \mathrm{Engl}$ J Med. 2005; 352(24):2477-86.

45. Reader D, Splett P, Gunderson EP. Diabetes Care and Education Dietetic Practice Group. Impact of gestational diabetes mellitus nutrition practice guidelines implemented by registered dietitians on pregnancy outcomes. J Am Diet Assoc. 2006; 106(9):1426-33. doi:10.1016/j.jada.2006.06.009.

46. Walker M. Breastfeeding with diabetes: yes you can! J Hum Lact. 2006; 22 (345): 345-6.

47. Reader D, Franz MJ. Lactation, diabetes and nutrition recommendations. Curr Diab Rep. 2004; 4(5):370-6.

Recebido em: 16/1/2008

Versão final reapresentada em: 22/8/2008

Aprovado em: 10/6/2009 
